Faculdade

de Ciências Econômicas UFRGS
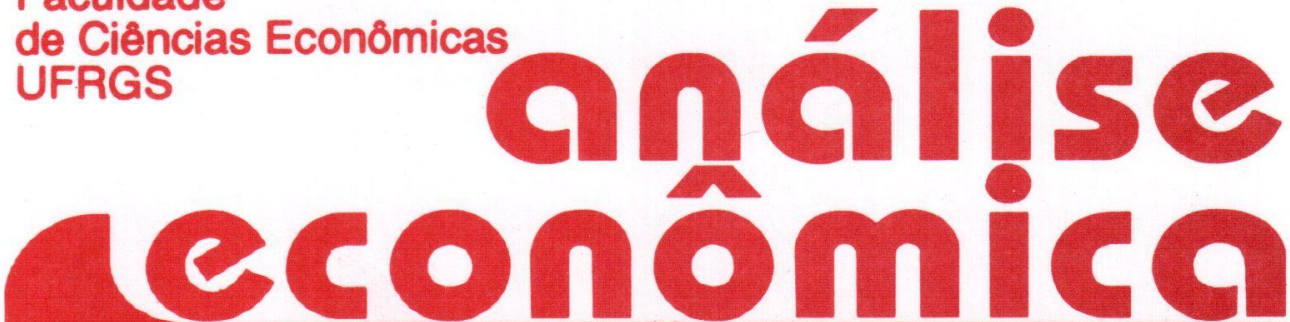

- EQUILIBRIO, PROGRESSO TÉCNICO E DESIGUALDADES REGIONAIS

Carlos Roberto Azzoni

- DESENVOLVIMENTO POLARIZADO E DESEQUILÍBRIOS REGIONAIS Nali de Jesus de Souza

- OS NOVOS CLÁSSICOS E O MÉTODO Carlos Magno Lopes

- ECONOMLAS DE MERCADO E DEMANDA EFETIVA Gilberto Tadeu Lima

- MEASURES OF CAPACITY UTILIZATION Marcelo S. Portugal

- ASPECTOS DO CONTROLE EM UM MODELO DINÂMICO

Marat Rafikow

Pedro Augusto P. Borges

- A FIRMA EM UM AMBIENTE INFLACIONÁRIO

Carmen A.do V.C. Feijó

- CUSTOS E BENEFICIOS DA INTEGRAÇĀO REGIONAL Marco Antônio Montoya

- A REESTRUTURAÇÃO DA ECONOMIA MUNDIAL Hoyêdo Nunes Lins

- O MERCADO COMO PROCESSO: A ABORDAGEM AUSTRÍACA

Fernando Caputo Zanella

- GARY BECKER: PRÊMIO NOBEL DE ECONOMIA DE 1992

Giácomo Babinotto Neto

LIVROS RECEBIDOS

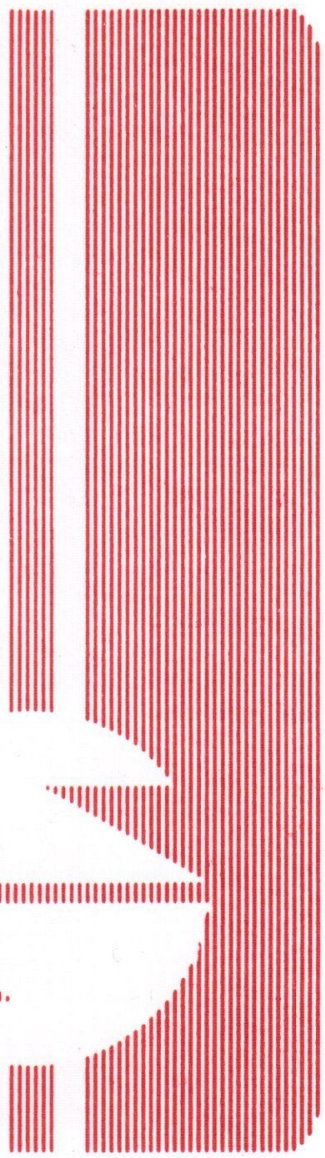


UNIVERSIDADE FEDERAL DO RIO GRANDE DO SUL Reitor: Prof. Hélgio Henrique Casses Trindade

FACULDADE DE CIENCIAS ECONOMICAS

Diretor: Prof. Pedro Cézar Dutra Fonseça

CENTRO DE ESTUDOS E PESQUISAS ECONOMICAS

Diretor: Prof. Roberto Pires Pacheco

DEPARTAMENTO DE CIENCIAS ECONÓMICAS

Chefe: Prof. Fernando Ferrari Filho

CURSO DE POS-GRADUAÇAOO EM ECONOMIA

Coordenador: Prof. Joăo Rogério Sanson

CURSO DE POS-GRADUAÇÂO EM ECONOMIA RURAL

Coordenador: Prof. Juvir Luiz Mattuella

CONSELHO EDITORIAL: Achyles Barcelos da Costa, Aray Miguel Feldens, Atos Freitas Grawunder, Carlos Augusto Crusius, Ernani Hickmann, Fernando Ferrari Filho, Joāo Rogério Sanson, Juvir Luiz Mattuella, Marcelo Savino Portunal, Maria Imilda da Costa e Silva, Nali de Jesus de Souza, Nuno Renan Lopes de Figueiredo Pinto, Otília Beatriz Kroeff Carrion, Otto Guilherme Konzen, Paulo Alexandre Sphor, Pedro Cezar Dutra Fonseca, Reinaldo I gnacio Adams, Roberto Camps Moraes, Valter José Stülp, Yeda Rorato Crusius, David Garlow (Wharton Econometrics Forecasts Association, E.U.A.), Edgar Augusto Lanzer (UFSC), Eleutério F. S. Prado (USP), Fernando Holanda Barbosa (FGV/RJ), Gustavo Franco (PUC/RJ), Joaquim Pinto de Andrade (UnB), Juan H. Moldau (USP), Werner Baer (Univ. de lllinois, E.U.A.).

COMISSĀO EDITORIAL: Atos Freitas Grawunder, Pedro Cezar Dutra Fonseca, Reinaldo Ignacio Adams e Roberto Camps Moraes.

EDITOR: Prof. Nali de Jesus de Souza

SECRETARIA: Maria Ivone de Mello (normalizaçāo), Vanete Ricacheski (revisão de textos).

FUNDADOR: Prof. Antônio Carlos Santos Rosa

Os materiais publicados na revisța Análise Econômica sāo da exclusiva responsabilidade dos autores. E permitida a reprodıçāo total ou parcial dos trabalhos, desde que seja citada a fonte.

Aceita-se permuta com revistas congêneres. Aceitam-se, também, livros para divulgaçāo, elaboraçāo de resenhas ou recensōes.

Toda correspondência, material para publicação (vide normas na terceira capa), assinaturas e permutas devem ser dirigidos ao seguinte destinatário:

\author{
PROF. ROBERTO CAMPS MORAES \\ Revista Análise Económica \\ Av. Joāo Pessoa, 52 \\ CEP 90040-000 - PORTO ALEGRE (RS), BRASIL \\ Telefones: (051) 228-1633, ramal 3440
}

Fax: (051) 225-1067 


\title{
ALGUNS ASPECTOS DO CONTROLE EM UM MODELO DINÂMICO DE OBJETOS DE PRODUÇÃO DE INTERAÇÕES CONFORME UMA FUNCIONAL QUADRÁTICA
}

\author{
Marat Rafikov* \\ Pedro Augusto P. Borges ${ }^{* *}$
}

\section{SINOPSE}

Neste artigo são considerados dois tipos de controle em relação ao sistema de dois objetos de interação de produçāo, em que um destes objetos produz uma mercadoria para aumentar os bens de capital e 0 outro prodiz $z \mathrm{um}$ produto final, a fim de suprir a jemanda dada. Inicialmente, determina-se - regime màis econômico de funcionamento do sistema, na condiçáo de conservação da capacidade produtiva do mesmo durante longo tempo. Posteriormente, considera-se o problema de estabilizaçăo do desenvolvimento do sistema de objetos de produção em relação à trajetória programada de desenvolvimento. O segundo problema resolve-se através do método de programação dinâmica. Como funcional objetivo foi usada uma funcional quadrática, que admite desvios das coordenadas espaciais e funçōes de controle do regime programático.

\section{INTRODUÇÃO}

Na moderna teoria do controle, distinguem-se problemas de dois tipos: o controle programácico e o contrule com realimentação.

No primeiro caso, o controle (6timo ou quase-6timo) é feito na forma de uma função do tempo com as condiçōes iniciais e as condiçōes finais concretas, isto $\epsilon$, $u=u(t)$. No segundo caso, o controle $e$ feito no instante $t$ como uma função do tempo e das variáveis-estado, isto $\varepsilon, \mathrm{u}=\mathrm{u}(\mathrm{x}, \mathrm{t})$. A realização destes dois tipos de controle são diferentes.

O controle programático é realizado através de um mecanismo programático de relogio, ou de um horário, conforme uma lei antecipada que é uma função do tempo. Este controle não reage contra desvios possíveis do estado do objeto

* Professor visitante da Universidade de ljuf,RS,PhD pelo Instituto Superior de Aviação de Kazan (Russia), bolsista da FAPERGS.

** Professor da Universidade de Ijuí, RS, M.Sc. pela UNICAMP.

Cód. AEA

Palavras-Chave:

213

Programaçáo dinâmica, Controle ótimo 
controlado em relação a um estado desejável, ideal. O controle com realimentação é realizado através de um regulador que, com base nos resultados das medidas dos valores reais das variáveis-estado, pode criar um sinal mediante o qual funciona um mecanismo de controle ou admite-se a decisão de controle. Ambos os problemas são de interações. Em problemas de controle econômico, existem estes dois tipos. Por exemplo, para investir em algum ramo da indústria, existe um programa de investimentos para cada momento do tempo. Isto é realizado através de um controle programático de investimentos. Mas devido a mudanças de condiçóes de funcionamento do sistema é necessário extinguir estes desvios, restabelecendo o sistema ao regime programático através de acréscimos ou retiradas de verbas correntes.

\section{O MODELO DINÂMICO DE UM SISTEMA DE OBJETOS DE PRODUÇÃO}

Consideraremos um sistema de dois objetos de produção. Um deles produz um produto usado no sistema como bens de capital. O outro produz uma mercadoria para usar fora do sistema. Sistemas deste tipo existem na realidade. Por exemplo, a indústria de máquinas agrícolas e a produção agrícola, ou a produção de máquinas para tecelagem e uma unidade de fabricação de tecidos. Através de modelos semelhantes pode-se modelar a reprodução de gado. Neste caso, o primeiro objeto modela a reprodução dos animais reprodutores e o segundo, a produção de gado para corte.

Se a demanda final é conhecida, então temos interesse no regime de funcionamento do sistema, quando a produção do primeiro objeto se realiza de forma a somente abasiecer a necessidade da demanda final e renovar o capital desgastado. Este regime de funcionamento é possível apenas para determinados valores das condiçôes iniciais do primeiro objeto. Se as condições iniciais reais são maiores que estes valores, então se observa o crescimento excessivo da capacidade do primeiro objeto. Se as condições iniciais reais são menores que estes valores, então se observa a diminuição da capacidade do primeiro objeto até a extinção inteira do objeto. durante um prazo finito.

O inodelo matemático do sistema cie dois objetos de interação tem a forma:

d $\phi_{1}$

$\overline{\mathrm{dt}}=\mathrm{u}_{1}-\alpha_{1} \phi_{1}$

$\mathrm{d} \phi 2$

$-u_{2}-\alpha_{2} \phi_{2}$

dt

$\mathrm{d} \phi_{\mathrm{n} 1}$

$-=\mathrm{U}_{1}-\mathrm{u}_{1}$

$\mathrm{dt}$ 


$$
\begin{aligned}
& \frac{\mathrm{d} \phi_{\mathrm{n} 2}}{\mathrm{dt}}=\mathrm{U}_{2}-\mathrm{u}_{2} \\
& \phi_{1}=\mathrm{k}_{1} \mathrm{x}_{1} \\
& \phi_{2}=\mathrm{k}_{2} \mathrm{x}_{2} \\
& \phi_{\mathrm{n} 1}=\tau_{1} \mathrm{u}_{1} \\
& \phi_{\mathrm{n} 2}=\tau_{2} \mathrm{u}_{2} \\
& \mathrm{x}_{1}=\mathrm{U}_{1}+\mathrm{U}_{2} \\
& \mathrm{x}_{2}=\overline{\mathrm{x}}
\end{aligned}
$$

onde :

$x_{i}$ é a produção do i-ésimo objeto no instante t;

$\phi_{i} \quad$ é a quantidade de bens do capital do i-ésimo objeto no instante t;

$\phi_{\text {ni }}$ é a quantidade do estoque de bens de capital do i-ésimo objeto que ainda não funciona para a produção;

ui é o fluxo da introdução em funcionamento de bens do capital no i-ésimo objeto no instante $t$;

$\mathrm{U}_{\mathrm{i}}$ é o fiuxo de bens de capital adquiridos para fins de uso do i-ésimo objeto no instante t;

$\overline{\mathrm{x}} \quad$ é a demanda final do produto do segundo objeto no instante $\mathrm{t}$;

$\mathrm{k}_{\mathrm{i}}$ é o coeficiente de capacidade do capital do i-ésimo objeto no instante $\mathrm{t}$;

$\alpha_{i}$ é o coeficiente de desgaste do capital no i-ésimo objeto no instante t;

$\tau_{i}$ é o coeficiente que caracteriza a duração do prazo da assimilação de estoques do capital do i-ésimo objeto no instante t;

1 Este modelo foi proposto em Sirazetdinov (1972) e foi modificado em Rafikov (1976). Uma diferença deste modelo para o modelo ve Leontief (1951) é que o modelo admite os processos de assimllaçáo e desgastes do capital do sistema. 
No nosso caso, os coefícientes $\mathrm{k}_{\mathrm{i}}, \alpha_{\mathrm{i}}, \tau_{\mathrm{i}}$, são constantes e conhecidos.

No sisterna (1), as duas primeiras equações descrevem o desenvolvimento da capacidade de cada objeto. As outras duas descrevem o processo da assimilação de bens de capital de cada objeto. As quatro seguintes modelam as suposiçóes necessárias de que a produção é proporcional aos bens de capital de cada objeto e o fluxo da introdução em funcionamento de bens do capital é proporcional à quantidade de bens de capital de cada objeto que ainda não funcionam para a produção. As duas últimas equações descrevem a distribuiçăo da produção de cada objeto.

Reescrevemos o sistema (1) de forma mais compacta:

$$
\begin{aligned}
& \frac{\mathrm{d} \phi_{1}}{\mathrm{dt}}=-\alpha_{1} \phi_{1}+\frac{1}{\tau_{1}} \phi_{\mathrm{n} 1} \\
& \frac{\mathrm{d} \phi_{2}}{\mathrm{dt}}=-\alpha_{2} \phi_{2}+\frac{1}{\tau_{2}} \phi_{\mathrm{n} 2} \\
& \frac{\mathrm{d} \phi_{\mathrm{n} 1}}{\mathrm{dt}}=\frac{-1}{\tau_{1}} \phi_{\mathrm{n} 1}+\mathrm{U}_{1} \\
& \frac{\mathrm{d} \phi_{\mathrm{n} 2}}{\frac{-1}{\mathrm{dt}}=\frac{-1}{\tau_{2}} \phi_{\mathrm{n} 2}+\mathrm{U}_{2}} \\
& \phi_{1}=\mathrm{k}_{1} \mathrm{x}_{1} \\
& \phi_{2}=\mathrm{k}_{2} \mathrm{X}_{2} \\
& \phi_{1} \\
& \frac{\mathrm{k}_{1}}{2}=\mathrm{U}_{1}+\mathrm{U}_{2} \\
& \mathrm{x}_{2}=\overline{\mathrm{x}}
\end{aligned}
$$

O desenvolvimento deste sistema determina-se por inteiro ao sistema de equações (1) ou (2) e condiçōes iniciais

$\phi_{\mathrm{i}(0)}=\phi_{\mathrm{i} 0}$

$$
\phi_{\text {ni }(0)}=\phi_{\text {nio }}
$$




\section{A SOLUÇÃO DO PROBLEMA DE CONTROLE PROGRAMÁTICO}

Seja

$$
\overline{\mathrm{x}}=\overline{\mathrm{x}}^{*}(\mathfrak{t})
$$

uma função de $t$ conhecida, contínua e derivável duas vezes. Explicitando $U_{2}$ da quarta equação do sistema (2) e substituindo $\phi_{\mathrm{n} 2}$ pela expressão

$$
\left(\frac{\mathrm{d} \phi_{2}}{\mathrm{dt}}+\alpha_{2} \phi_{2}\right) \tau_{2}
$$

que foi obtida da segunda equação do sistema (2) podemos encontrar a variável de controle $\mathrm{U}_{2^{*}}$ que é necessário para o desenvolvimento do segundo objeto em concordância com a demanda final:

$$
\mathrm{U}_{2}{ }^{*}=\mathrm{k}_{2} \tau_{2} \frac{\mathrm{d}^{2} \overline{\mathrm{x}}^{*}}{\mathrm{dt}^{2}}+\left(\alpha_{2} \tau_{2}+1\right) \mathrm{k}_{2} \frac{\mathrm{d}^{*}{ }^{*}}{\mathrm{dt}}+\alpha_{2} \mathrm{k}_{2} \overline{\mathrm{x}}^{*}
$$

Fazendo algumas transformações obvias com as equações do sistema (2), obtemos:

$$
\begin{aligned}
& \frac{\mathrm{d} \phi_{1}}{\mathrm{dt}}=-\alpha_{1} \phi_{1}+\frac{1}{\tau_{1}} \phi_{\mathrm{n} 1} \\
& \frac{\mathrm{d} \phi_{\mathrm{n} 1}}{\mathrm{dt}}=\frac{1}{\mathrm{k}_{1}} \phi_{1}-\frac{1}{\tau_{1}} \phi_{\mathrm{n} 1}-\mathrm{U}_{2} *
\end{aligned}
$$

onde a função $\mathrm{U}_{2}^{*}$ é determinada através da expressão (4). Reescrevemos o sistema (5) na forma matricial:

$$
F^{\prime}=A F+V
$$

onde

$$
\mathrm{F}:=\left[\begin{array}{c}
\phi_{1} \\
\phi_{\mathrm{n} 1}
\end{array}\right] ; \quad \mathrm{V}=\left[\begin{array}{c}
0 \\
-\mathrm{U}^{*}
\end{array}\right] ; \quad \mathrm{A}=\left[\begin{array}{cc}
-\alpha_{1} & 1 / \tau_{1} \\
1 / \mathrm{k}_{1} & -1 / \tau_{1}
\end{array}\right]
$$


A solução geral do sistema (6) pode ser escrita na forma:

$$
\mathrm{F}=\mathrm{e}^{\mathrm{At}} \mathrm{C}+\mathrm{e}^{\mathrm{At}} \int \mathrm{e}^{-\mathrm{At}} \mathrm{Vdt},
$$

onde $\mathrm{e}^{\mathrm{At}}$ conforme definição é uma matriz:

$$
\mathrm{e}^{\mathrm{At}}=\sum_{n=1}^{\infty} \frac{1}{\mathrm{n} !} \mathrm{A}^{n} \mathrm{t}^{n}
$$

onde Cé um vetor-coluna $\left[\begin{array}{l}\mathrm{C}_{1} \\ \mathrm{C}_{2}\end{array}\right]$.

A solução geral de (7) consiste de duas partes. Uma delas $\mathrm{e}^{\mathrm{At}} \mathrm{C}$ caracteriza o desenvolvimento próprio do primeiro objeto (a solução geral da equação homogênea associada). A outra parte

$$
\mathrm{e}^{\mathrm{At}} \int \mathrm{e}^{-\mathrm{At}} \mathrm{Vdt} \text {, }
$$

caracteriza o desenvolvimento forçado que é condicionado ao vetor de controle $\mathrm{V}$. Obviamente, não é necessário desenvolver demais o primeiro objeto, mas o suficiente para desenvolver o segundo e compensar os desgastes de ambos. Deste ponto de vista, o regime mais favorável é quando $\mathrm{C}=0$. Então o regime procurado de desenvolvimento do primeiro objeto é encontrado pela formula

$$
F^{*}=e^{A t} \int e^{-A t} V d t \text {. }
$$

O regime (8) foi encontrado aciocinando-se em relação ao desenvolvimento do primeiro objeto, quando o segundo objeto produz um produto do tamanho necessário. A rigor, não podemos chamar este regime de ótimo, pois como e de praxe na teoria do controle ótimo, é difícil determinar a funcional mínima conforme este regime.

Assim, as funções

$$
\phi_{2}^{*}, \phi_{2}^{*}, \phi_{n 1} * \text { e } \phi_{n 2}{ }^{*}
$$

que se obtêm atravês das expressões

$$
\begin{aligned}
& {\left[\begin{array}{c}
\phi^{*} 1 \\
\phi^{*}{ }_{n}
\end{array}\right]=\mathrm{e}^{\mathrm{At}} \int \mathrm{e}^{-\mathrm{At}}\left[\begin{array}{c}
0 \\
-\mathrm{U}_{2}^{*}
\end{array}\right] \mathrm{dt},} \\
& \phi_{2}^{*}=\mathrm{k}_{2} \overline{\mathrm{x}}^{*} \quad \text { e } \quad \phi_{\mathrm{n} 2}^{*}=\tau_{2}\left(\mathrm{k} 2 \frac{\mathrm{d} \overline{\mathrm{x}}}{\mathrm{dt}}+\alpha_{2 \mathrm{k} 2 \overline{\mathrm{x}})}\right.
\end{aligned}
$$


determinam tal desenvolvimento programático do sistema considerado quando o segundo objeto produz a quantidade necessária do produto final e o primeiro objeto funciona somente para o abastecimento das necessidades do segundo objeto e também para compensação de desgastes de ambos. O desenvolvimento programático é realizado através de controle, ou seja, dos programas:

$$
\begin{aligned}
& U_{1}^{*}(t)=\frac{1}{k_{1}} \phi_{1}^{*}-U_{2}^{*} \\
& \mathrm{U}^{*} z(\mathrm{t})=\mathrm{k}_{2} \tau_{2} \frac{\mathrm{d}^{2} \overline{\mathrm{x}}^{*}}{\mathrm{dt}^{2}}+\left(\alpha_{2} \tau_{2}+1\right) \mathrm{k}_{2} \frac{\mathrm{d} \overline{\mathrm{x}}^{*}}{\mathrm{dt}}+\alpha_{2} \mathrm{k}_{2} \overline{\mathrm{x}} *
\end{aligned}
$$

\section{O SISTEMA DINÂMICO EM DESVIOS}

De modo geral, o desenvolvimento real do sistema é diferente do desenvolvimento programático (9) e (10). O estado do sistema de objetos pode ser representado como a soma do desenvolvimento programático e dos desvios do programa, isto é:

$$
\begin{aligned}
& \phi_{1}=\phi^{*}{ }_{1+\mathrm{y}_{1}} \\
& \phi_{2}=\phi^{*}{ }_{2+\mathrm{y}_{2}} \\
& \phi_{\mathrm{n} 1}=\phi^{*}{ }_{\mathrm{n} 1+\mathrm{y}_{3}} \\
& \phi_{\mathrm{n} 2}=\phi_{\mathrm{n} 2+\mathrm{y}_{4}}^{*} \\
& \mathrm{U}_{1}=U_{1}^{*}+\Delta \mathrm{U}_{1} \\
& \mathrm{U}_{2}=U^{*}{ }_{2}+\Delta \mathrm{U}_{2}
\end{aligned}
$$

onde y1 e y2 são os desvios entre os valores de bens de capital reais e os valores de bens de capital do regime programático no primeiro e segundo objetos respectivamente; y3 e y4 são os desvios entre os valores reais de estoques de capital não utilizados para produção e os valores programáticos destes estoques; $\Delta U_{1} \mathrm{e}$ $\Delta \mathrm{U}_{2}$ são desvios entre os valores reais das variáveis de controle e os valores programáticos.

As funções (9) e (10) que caracterizam o regime programático satisfazem o sistema (2), isto e: 


$$
\begin{aligned}
& \frac{\mathrm{d} \phi_{1}^{*}}{\mathrm{dt}}=-\alpha_{1} \phi_{1}+\frac{1}{\tau_{1}} \phi_{\mathrm{n} 1}^{*} \\
& \frac{\mathrm{d} \phi_{2}^{*}}{\mathrm{dt}}=-\alpha_{2} \phi_{2}+\frac{1}{\tau_{2}} \phi_{\mathrm{n} 2}^{*} \\
& \frac{\mathrm{d} \phi_{\mathrm{n} 1}^{*}}{\mathrm{dt}}=\frac{-1}{\tau_{1}} \phi_{\mathrm{n} 1}^{*}+\mathrm{U}_{1}^{*} \\
& \frac{\mathrm{d} \phi_{\mathrm{n} 2}^{*}}{\mathrm{dt}}=\frac{-1}{\tau_{1}} \phi_{\mathrm{n} 2}^{*}+U_{2}^{*} \\
& \frac{1}{\mathrm{k}_{1}} \phi_{1}^{*}=\mathrm{U}_{1}^{*}+U_{2}^{*}
\end{aligned}
$$

Subtraindo as equações do sistema (12) das respectivas equações do sistema (2) e admitindo as expressões (11), obtemos o sistema de equaçōes em desvios:

$$
\begin{aligned}
& \frac{d y_{1}}{d t}=-\alpha_{1} y_{1}+\frac{1}{\tau_{1}} y_{3} \\
& \frac{d y_{2}}{d t}=-\alpha_{2} y_{2}+\frac{1}{\tau_{2}} y_{4} \\
& \frac{d_{3}}{d t}=-\frac{1}{\tau_{1}} y_{3}+\Delta U_{1} \\
& \frac{d y_{4}}{d t}=-\frac{1}{\tau_{2}} y_{4}+\Delta U_{2} \\
& \frac{1}{k_{1}} y_{1}=\Delta U_{1}+\Delta U_{2}
\end{aligned}
$$


Substituindo a última equação na quarta equação do sistema (13) e usando a forma matricial, obtemos:

$$
Y^{\prime}=A Y+B \Delta U_{1}
$$

onde

$$
\mathrm{Y}=\left[\begin{array}{l}
\mathrm{Y}_{1} \\
\mathrm{Y}_{2} \\
\mathrm{Y}_{3} \\
\mathrm{Y}_{4}
\end{array}\right] ; \quad \mathrm{A}=\left[\begin{array}{llll}
-\alpha_{1} & 0 & 1 / \tau_{1} & 0 \\
0 & -\alpha_{2} & 0 & 1 / \tau_{2} \\
0 & 0 & -1 / \tau_{1} & 0 \\
1 / \mathrm{k}_{1} & 0 & 0 & -1 / \tau_{2}
\end{array}\right] ; \mathrm{B}=\left[\begin{array}{c}
0 \\
0 \\
1 \\
-1
\end{array}\right]
$$

e $\Delta U_{1}$ é uma função escalar de controle.

As condiçōes iniciais têm a forma:

$$
\mathrm{Y}(0)=\left[\begin{array}{l}
\mathrm{y}_{1}(0) \\
\mathrm{y}_{2}(0) \\
\mathrm{y}_{3}(0) \\
\mathrm{y}_{4}(0)
\end{array}\right]
$$

onde :

$$
\begin{aligned}
& \mathrm{y}_{1}(0)=\phi_{1}(0)-\phi_{1}^{*}(0) \\
& \mathrm{y}_{2}(0)=\phi_{2}(0)-\mathrm{k}_{2}{ }_{\mathrm{x}}^{*} \\
& \mathrm{y}_{3}(0)=\phi_{\mathrm{n} 1(0)}-\phi_{\mathrm{n} 1(0)}^{*} \\
& \mathrm{y}_{4}(0)=\phi_{\mathrm{n} 2}(0)-\phi_{\mathrm{n} 2(0)}^{*}
\end{aligned}
$$

A funcional objetivo é dada por:

$$
\mathrm{I}\left[\Delta \mathrm{U}_{1}\right]=\frac{1}{2} \int_{0}^{\infty}\left(\mathrm{Y}^{\mathrm{T}} \mathrm{QY}+\Delta \mathrm{U}_{1} 2\right) \mathrm{dt} .
$$

onde $\mathrm{Q}$ é uma matriz de pesos simétrica semidefinida positiva.

A funcional objetivo (16) contém a soma dos qũadrados de desvios entre os valores reais das variáveis e os valores do regime programático. Minimizando esta funcional, podemos determinar uma fungao de controle otima $\Delta \mathrm{U}_{1}$ que diminui os desvios das variáveis com gastos minimais. 


\section{A FORMULAÇÃO DO PROBLEMA DE CONTROLE ÓTIMO}

Formularemos o problema de desvios mínimos de regime programático como o problema de síntese de controle otimo: ${ }^{2}$ encontrar um controle otimo que fornece um valor mínimo da funcional (16) e transfere o sistema (14) do estado inicial (15) para um estado final:

$$
\mathrm{Y}(\infty)=0
$$

Se encontramos o controle $\Delta U 1$ de um conjunto aberto, então o controle ótimo linear incôgnito pode ser determinado pela expressão:

$$
\Delta U_{1}=-B^{T} S Y
$$

onde S é uma matriz simétrica de coeficientes ótimos que é a solução da equação matricial não-linear:

$$
-S A \cdot A^{T} S+S B B^{T} S-Q=0
$$

Em (19) a matriz Q é dada.

Se a matriz $\mathrm{S}$ tem as componentes

$$
S=\left[\begin{array}{llll}
S_{11} & S_{12} & S_{13} & S_{14} \\
S_{12} & S_{22} & S_{23} & S_{24} \\
S_{13} & S_{23} & S_{33} & S_{34} \\
S_{14} & S_{24} & S_{34} & S_{44}
\end{array}\right]
$$

então a expressão (18) assume a forma:

$$
\Delta \mathrm{U}_{1}=-\mathrm{p}_{1} \mathrm{y}_{1}-\mathrm{p}_{2} \mathrm{y}_{2}-\mathrm{p}_{3} \mathrm{y}_{3}-\mathrm{p}_{4} \mathrm{y}_{4}
$$

onde $p_{i}=S_{i 3}-S_{i 4} \quad i=1,2,3,4$.

Geralmente usa-se $\mathrm{Q}$ como a matriz diagonal:

$$
\mathrm{Q}=\left[\begin{array}{llll}
\mathrm{Q}_{11} & 0 & 0 & 0 \\
0 & \mathrm{Q}_{22} & 0 & 0 \\
0 & 0 & \mathrm{Q}_{33} & 0 \\
0 & 0 & 0 & \mathrm{Q}_{44}
\end{array}\right]
$$

$\mathrm{Se} \mathrm{Q}_{11}, \mathrm{Q}_{22}, \mathrm{Q}_{33}, \mathrm{Q}_{44}$ são dados, podemos encontrar $\mathrm{S}_{\mathrm{i} 4}, \mathrm{~S}_{\mathrm{i} 3}$

2 Bellman (1957), Athans and Falb (1966), Letov (1969). 
i=1,2,3,4 da equação (19) e depois determinar a variável de controle conforme (20). Sabendo a variável de controle $\Delta U_{1}$ da equação (14) obtemos o processo transitório que descreverá o desenvolvimento do sistema em desvios.

\section{O PROBLEMA DA ESCOLHA DOS COEFICIENTES DE PONDERABILIDADE DA FUNCIONAL OBJETIVO}

Precisa-se notar que, para valores diferentes da matriz de coeficientes de ponderabilidade $\mathrm{Q}_{\mathrm{ii}}$, temos diferentes trajetorias, ou seja, a variação de valores das componentes da matriz $Q$ essencialmente influi na qualidade do processo transitório. Então, supondo-se valores diferentes dos coeficientes de ponderabilidade, obtemos diferentes valores da funcional otimizada e diferentes trajetórias de desenvolvimento. A análise da influência dos coeficientes de ponderabilidade da funcional otimizada ao processo da transição $e$ um problema singular geralmente bastante complicadú.

Um caso particular do nosso problema é quando a assimilação de estoques do capital acontece instantaneamente, i.é: se

$$
\phi_{\mathrm{ni}}=0, \quad \tau_{\mathrm{ni}} \approx 0 \quad \text { para } \mathrm{n}=1,2,
$$

podemos obter as expressões analíticas que descrevem a dependência entre as características do processo de transição e os coeficientes de ponderabilidade da funcional otimizada.

Consideraremos este caso minuciosamente. No caso

$\phi_{n i}=0, \quad \tau_{i}=0 \quad$ temos que $u_{i}=U_{i}, \quad i=1,2$

Então n sistema (1) tem a forma

$$
\begin{aligned}
& \frac{\mathrm{d} \phi_{\mathrm{i}}}{\mathrm{dt}}=\mathrm{U}_{\mathrm{i}}-\alpha_{\mathrm{i}} \phi_{\mathrm{i}} \\
& \phi_{\mathrm{i}}=\mathrm{k}_{\mathrm{i}} \mathrm{x}_{\mathrm{i}} \quad \mathrm{i}=1,2 \\
& \mathrm{x}_{1}=\mathrm{U}_{1}+\mathrm{U}_{2} \\
& \mathrm{x}_{2} \stackrel{\doteq}{=} \overline{\mathrm{x}}(\mathrm{t})
\end{aligned}
$$

A equação em desvios tem a forma (19), em que 


$$
A=\left[\begin{array}{cc}
-\alpha_{1} & 0 \\
1 / k_{1} & -\alpha_{2}
\end{array}\right] ; \quad Y=\left[\begin{array}{l}
y_{1} \\
y_{2}
\end{array}\right] ; \quad B=\left[\begin{array}{c}
1 \\
-1
\end{array}\right]
$$

As condições iniciais tem a forma:

$$
Y(0)=\left[\begin{array}{l}
y_{1}(0) \\
y_{2}(0)
\end{array}\right]
$$

onde

$$
\begin{aligned}
& \mathrm{y}_{1}(0)=\phi_{1}(0)-\phi 1 *(0) \\
& \mathrm{y}_{2}(0)=\hat{\varphi_{2}}(0)-\mathrm{k}_{2} \overline{\mathrm{x}}^{*}
\end{aligned}
$$

A matriz $Q$ dos coeficientes de ponderabilidade da funcional objetivo (16) tem componentes:

$$
\mathrm{Q}=\left[\begin{array}{ll}
\mathrm{Q}_{11} & 0 \\
0 & \mathrm{Q}_{22}
\end{array}\right]
$$

A matriz simétrica $S$ tem componentes:

$$
S=\left[\begin{array}{ll}
S_{11} & S_{12} \\
S_{12} & S_{22}
\end{array}\right]
$$

A variável de controle 6tima (18) depende apenas dos desvios y1 e y2:

$$
\Delta \mathrm{U} 1=-\mathrm{p}_{1} \mathrm{y}_{1}-\mathrm{p}_{2} \mathrm{y}_{2}
$$

onde

$$
\mathrm{p}_{\mathrm{i}}=\mathrm{S}_{\mathrm{ii}}-\mathrm{S}_{\mathrm{i} 2} ; \mathrm{i}=1,2
$$

Levando a variável de controle (27) em (14), obtemos um sistema de duas equaçōes diferenciais lineares: 


$$
\begin{aligned}
& \frac{d y_{1}}{d t}=-\left(\alpha_{1}+p_{1}\right) y_{1}-p_{2} y_{2} \\
& \frac{d y_{2}}{d t}=\left(\frac{1}{k_{1}}+p_{1}\right) y_{1}+\left(p_{2}-\alpha_{2}\right) y_{2}
\end{aligned}
$$

Reescrevemos o sistema (29) na forma de uma equação diferencial de segunda ordem:

$$
T^{2} \frac{d^{2} y_{1}}{d t^{2}}+2 \xi T \frac{d y_{1}}{d t}+y_{1}=0
$$

onde

$$
\begin{aligned}
& \mathrm{T}=\frac{1}{\sqrt{\alpha_{1} \alpha_{2}-\alpha_{1} p_{2}+\alpha_{2} p_{2}+p_{2} / k_{1}}} \\
& \xi=\frac{T}{2}\left(\alpha_{1}+\alpha_{2}+p_{1}-p_{2}\right) .
\end{aligned}
$$

A equação (30), do ponto de vista da teoria do controle, é uma equação do elo dinâmico ou oscilatório. Os parâmetros do elo chiamam-se: a constante de tempo $\mathrm{T}$ e o coeficiente relativo de amortecimento $\xi$. São deterniinados respectivamente pelas expressões (31) e (32). Destas podemos explicitar p1 e p2 como as funções dos parâmetros $\mathrm{Te} \xi$.

Depois de algumas transformações obtemos:

$$
\frac{\mathrm{T}^{2}\left(\alpha^{2}-\frac{\alpha_{2}+\alpha_{1}}{\mathrm{k}_{1}}\right)-2 \xi \mathrm{T}\left(\alpha_{1}-\frac{1}{\mathrm{k}_{1}}\right)+1}{\mathrm{~T}^{2}\left(\frac{1}{\mathrm{k}_{1}}-\alpha_{1}+\alpha_{2}\right)} ;
$$




$$
\mathrm{p} 2=\frac{\mathrm{T}^{2} \alpha^{2}-2 \xi T \alpha_{2}+1}{T^{2}\left(\frac{1}{\mathrm{k}_{1}}-\alpha_{1}+\alpha_{2}\right)}
$$

Determinando $\mathrm{p}_{1}$ e $\mathrm{p}_{2}$ das formulas (33), podemos obter as componentes da matriz $S$ das expressões (19) e (21):

$$
\begin{aligned}
& S_{22}=\frac{2 \alpha_{2} p_{2}-p_{1} p_{2}}{2 \alpha_{2}-\frac{1}{k_{1}}} \\
& S_{11}=p_{1}+S_{12} \\
& S_{12}=p_{2}+S_{22}
\end{aligned}
$$

Da equação (19) também podemos obter os valores dos coeficientes de ponderabilidade da funcional ubjetivo:

$$
\begin{aligned}
& \mathrm{Q}_{11}=2\left(\alpha_{1} \mathrm{~S}_{11} \frac{\mathrm{S}_{12}}{\mathrm{k}_{1}}\right)+\mathrm{p}_{12} \\
& \mathrm{Q}_{22}=2 \alpha_{2} \mathrm{~S}_{22}+\mathrm{p}_{2} 2
\end{aligned}
$$
colocado:

Então podemos organizar o seguinte algoritiino da resolução do problema

a) Determinamos os parâmetros $T$ e $\xi$ do processo de transição, ou seja, a qualidade do processo de transição.

b) Verificamos que estes parâmetros $\mathrm{T}$ e $\xi$ satisfazem a condição de que a matriz dos coeficientes de ponderabilidade é semidefinida positiva. Isto $\epsilon$, quando os parâmetros acima são definidos, conforme as formulas (34) e(35) determinamos Q11 e $Q_{22}$ e verificamos as condições

$\mathrm{Q}_{11} \geq 0 \quad$ e $\mathrm{Q}_{22} \geq 0$.

c) Das expressões (33) calculamos os coeficientes de reforço p p e p2 da função de controle $\Delta \mathrm{U}_{1}$.

Sabendo p1 e p2, da fórmula (27) obtemos a função de controle ótimo $\Delta U_{1}$. d) Substituindo $\Delta U_{1}$ no sistema (14) e integrando-o podemos obter (se 
precisarmos) o processo de transição, isto é:

$$
\begin{aligned}
& y_{1}=y_{1}(t) \\
& y_{2}=y_{2}(t) .
\end{aligned}
$$

Conforme a colocação do problema, temos que

$$
\lim _{t \rightarrow \infty} \text { yi }(\mathfrak{t})=0
$$

mas especificando os parâmetros certos de $\mathrm{Te} \xi$ podemos fazer com que durante um prazo finito $y_{1} \mathrm{e}$ y 2 tenham valores proximos a zero.

Precisa-se notar que os coeficientes de reforço $p_{i}$ dependem somente dos parâmetros do modelo $\alpha_{i}$ e $k_{i} ; i=1,2$ e dos parâmetros do processo de transição $\xi$ e T e então são constantes para nosso problema durante todo o tempo de regulagem. Assim é suficiente determinar $\mathrm{p}_{1} \mathrm{e} \mathrm{p}_{2}$ iricialmente, e depois calcular $\Delta \mathrm{U}_{1}$ de acordo com os valores atuais de $\mathrm{y}_{1} \mathrm{e} \mathrm{y}_{2}$.

Gostaríamos de salientar que, no sistema dado, faltam equações que caracterizam os insumos. Na resolução deste problema, consideramos que os insumos são proporcionais à produção. Sabendo o nível da produção sempre podemos determinar os insumos através da fórmula:

$$
v_{i j}=a_{i j} x_{j} \quad i=1,2, \ldots S ; j=1,2
$$

onde $a_{i j}$ são os coeficientes de insumo-produto;

$\mathrm{v}_{\mathrm{ij}} \mathrm{s}$ ão a quantidade do insumo do tipo i necessário para o funcionamento do j-ésimo objeto no instante t;

S é a quantidade de tipos de insumo.

\section{EXEMPLO}

Consideraremos o sistema de dois objetos, em que o primeiro produz apenas máquinas e equipamentos para o outro e para sua propria utilização, enquanto que o segundo produz apenas um produto final, para suprir uma demanda dada. Semelhante modelo pode ser usado para modelar, por exemplo, um sistema que abranja um conjunto de fábricas que produzam alimentos (segundo objeto do sistema) e um conjunto de fábricas que produzam máquinas e equipamentos para a indústria alimentícia (primeiro objeto do sistema).

Seja a demanda final do segundo objeto especificado uma função linear do tempo:

$$
\overrightarrow{\mathrm{x}}^{*}=\mathrm{bt}+\mathrm{c},
$$


onde b, c são números dados.

Para suprir esta demanda final no segundo objeto, devem ser introduzidas máquinas e equipamentos em quantidade:

$$
\mathrm{U}^{*} 2=\mathrm{bk}_{2}+\alpha_{2 \mathrm{k}}(\mathrm{bt}+\mathrm{c})
$$

Então o desenvolvimento programático do primeiro objeto que corresponde a esta demanda final é determinado mediante a equação:

$$
\left.\frac{d \phi_{1}^{*}}{d t}=\frac{1}{\left(k_{1}\right.}-\alpha_{1}\right) \phi_{1}^{*}-b k_{2}-\alpha_{2} k_{2}(b t+c)
$$

e uma condição inicial

$$
\phi_{1} *(0)=\frac{b k_{2}+c \alpha_{2} k_{2}}{\left(\frac{1}{k_{1}}-\alpha_{1}\right)}+\frac{b \alpha_{2} k_{2}}{\left(\frac{1}{k_{1}}-\alpha_{1}\right)^{2}}
$$

No caso particular do nosso exemplo, o regime programático pode ser determinado de maneira mais fácil. Supondo que a solução particular correspondente ao regime programático deve ser uma função linear (pois a demanda final é função linear), procuramos esta solução na forma linear. Substituindo esta função na equação (38), obtemos:

$$
\phi_{1(t)}^{*}=\frac{b \alpha_{2} k_{2}}{\left(\frac{1}{k_{1}}-\alpha 1\right)} t+\frac{b \alpha_{2} k_{2}}{\left(\frac{1}{k_{1}}-\alpha 1\right)^{2}}+\frac{b k_{2}+c \alpha_{2} k_{2}}{\left(\frac{1}{k_{1}}-\alpha 1\right)}
$$

Se no momento inicial as condições iniciais não concordam com as iniciais programáticas, a saber:

$$
\begin{aligned}
& \phi_{1}(0)<\phi_{1}^{*}(0) \\
& \phi_{2}(0)<\mathrm{k}_{2} \overrightarrow{\mathrm{x}}^{*}(0)=\mathrm{k}_{2} \mathrm{ck}_{2},
\end{aligned}
$$

então existem os desvios das variáveis-estado: 


$$
\begin{aligned}
& \mathrm{y}_{1}=\phi_{1}-\phi_{1}^{*} \\
& \mathrm{y}_{2}=\phi_{2}-\mathrm{k}_{2} \overline{\mathrm{x}}^{*}
\end{aligned}
$$

Formularemos o seguinte problema: encontrar um controle otimo que transforme o sistema (29) do estado inicial:

$$
\begin{aligned}
& y_{1}(0)=\phi_{1}(0)-\frac{b k_{2}+c \alpha_{2} k_{2}}{\left(\frac{1}{k_{1}}-\alpha_{1}\right)}+\frac{b \alpha_{2} k_{2}}{\left(\frac{1}{k_{1}}-\alpha_{1}\right)^{2}} \\
& y_{2}(0)=\phi_{2}(0)-c k_{2}
\end{aligned}
$$

para um estado final:

$$
\mathrm{y}_{\mathrm{i}}(\infty)=0 \quad \mathrm{i}=1,2
$$

e que forneça um valor mínimo da funcional (16).

Usando os valores dos parâmetros do modelo da tabela 1 , resolvemos este problema conforme o algoritmo acima referido.

\section{TABELA 1. VALORES DOS PARÂMETROS DO MODELO}

\begin{tabular}{cccccc}
\hline$\alpha_{1}$ & $\mathrm{a}_{2}$ & $\mathrm{k}_{1}$ & $\mathrm{k}_{2}$ & $\mathrm{~b}$ & $\mathrm{c}$ \\
\hline 0,1 & 0,15 & 0,25 & 0,33 & 0,1 & 52 \\
\hline
\end{tabular}

Definimos $T=1,5 \mathrm{e} \xi=1$ e depois dos cálculos verificamos que estes valores satisfazem a condiçăo que a matriz Q e a semidefinida positiva. Então de (33) calculamos p1 e p2:

$$
\mathrm{p}_{1}=2,5858 ; \mathrm{p}_{2}=0,1483 \text {; }
$$

de (34) calculamos $S_{11}, S_{12}, S_{11}$ :

$$
S_{22}=0,0916 ; S_{12}=0,2399 ; S_{11}=2,8257 \text {; }
$$

e finalmente calculamos $Q_{11}, Q_{22}$ de (35):

$$
\mathrm{Q}_{11}=5,3321 ; \mathrm{Q}_{22}=0,0495 \text {. }
$$

Os 'valores $\mathrm{T} \xi$ e são válidos pois $\mathrm{Q}_{11}>0, \mathrm{Q}_{22}>0$. $\mathrm{O}$ controle 6timo para o problema formulado é:

$$
\Delta \mathrm{U}_{1}=2,5858 \mathrm{y}_{1}+0,1483 \mathrm{y}_{2} \text {. }
$$


Então a variável de controle $\Delta U_{2} e ́$ :

$$
\Delta \mathrm{U}_{2}=\frac{1}{\mathrm{k}_{1}} \mathrm{y}_{1}-\Delta \mathrm{U}_{1}=1,4142 \mathrm{y}_{1}-0,1483 \mathrm{y}_{2}
$$

Conforme as expressões (24) e (27) as funções $U^{*}{ }_{2}$ e $\phi^{*} 1$ que caracterizam o regime programático são lineares:

$$
\begin{aligned}
& U_{2}^{*}=0,00495 t+2,607 \\
& \phi_{1}^{*}=0,00127 t+0,6603
\end{aligned}
$$

A função $U^{*}{ }_{1}$ também é uma função linear:

$$
\mathrm{U}_{1}^{*}=\frac{1}{\mathrm{k}_{1}} \phi_{1}^{*}-\mathrm{U}_{2}=0,00013 \mathrm{t}+0,0342
$$

E definitivamente, um controle que admite ambos os regimes, programático e de regulagem, tem a forma:

$$
\begin{aligned}
& \mathrm{U}_{1}=\mathrm{U}_{1}^{*}+\Delta \mathrm{U}_{1}=0,00013 \mathrm{t}+0,0342+2,5858 \mathrm{y}_{1}+0,1483 \mathrm{y}_{2} \\
& \mathrm{U}_{2}=\mathrm{U}_{2}^{*}+\Delta \mathrm{U}_{2}=0,00495 \mathrm{t}+2,607+1,4142 \mathrm{y}_{1}-0,1483 \mathrm{y}_{2}
\end{aligned}
$$

\section{BIBLIOGRAFIA}

ATHANS, N. \& FALB, P. Optimal Control: an Introduction to the Theory and its Aplications. New York: Mc Graw.Hill, 1966.

BELLMANN, R. Dynamic Programming. New Jersey: Princeton University, 1957.

LEONTIEF, W.W. The Structure of American Economy, 1919-1939. New York: Fair-Lawn, Oxford University Press, 1951,2a. ed.

LETOV, A.M.Dinamica Poleta e Upravlenie. Moscou: Naúca, 1969. (Dinâmica de Vôo e o Controle). RAFIKOV, M.M. Optimalnoe Uprevlenie Sistemoi Vzaimodeistvuiuchik Proizvodstvennik Obhektov po Quatdratichnomu Criteriu Cachestva. In: Optimizacia Processov v Aviacionnoi Tecnike, Kazan: KAI, 1976. (Controle Ótimo de um Sistema de Objetos, de Produção com a Funcional Objetivo Quadrática).

SIRAZETDINOV T.K. Dinamitcheskaia Model Economitcheskogo Obhekta. Kazan: IVUZ, Aviacionnaia Tecnika, n.9, 1972. (Um Modelo Dinâmico do Objeto Econômico). 


\section{ABSTRACT \\ SOME CONTROL IN A DYNAMIC MODEL OF INTERACTING PRODUCTION OBJECTS WITH A QUADRATIC FUNCTIONAL FORM}

In this paper are considered two types of control for a given system of two interacting objects production, in wich one of this objects manufactures a merchandise to increase capital, and the other a final product in order to supply a given demand. At first is determinated the most economic working regime for the system and the condition of conserving it's working capacity for a long time. Then, it is considered the problem of optimal stabilization of the development of this two-object system in relation to the programmed trajectory of development. The second problem is solved through the method of dynamic programming. As the object functional is a quadratic funtional that admits deviations of the spatial coordinate and control functions for the programmed regime. 